\title{
magnetic resonance in endomyocardial fibrosis
}

\section{Petronella Samuels", Stephen Jermy",\#, Sulaiman Moosa ${ }^{\dagger}$ and Ntobeko A.B. Ntusi",t,*}

*Cape Universities Body Imaging Centre, Faculty of Health Sciences, University of Cape Town, Observatory, Cape Town, South Africa "Division of Biomedical Engineering, Department of Human Biology, Faculty of Health Sciences, University of Cape Town, Observatory, Cape Town, South Africa

tDivision of Cardiology, Department of Medicine, University of Cape Town and Groote Schuur Hospital, Observatory,

Cape Town, South Africa

\# Hatter Institute for Cardiovascular Research in Africa, Faculty of Health Sciences, University of Cape Town, Cape Town, South Africa

Address for correspondence:

Prof Ntobeko Ntusi

Chair and Head: Department of Medicine

University of Cape Town and Groote Schuur Hospital

J46.53 Old Main Building

Groote Schuur Hospital

Main Road

Observatory

7925

South Africa

Email:

ntobeko.ntusi@uct.ac.za

\section{INTRODUCTION}

Endomyocardial fibrosis (EMF) is an idiopathic disorder of the tropical and subtropical regions of the world, considered endemic in tropical and subtropical Africa and is characterised by development of restrictive cardiomyopathy. First described in 1948 by John Davies in Uganda, it is typically a disease associated with rural poverty and is typified by fibrosis of the apical endocardium of the right ventricle (RV), left ventricle (LV), or both. The clinical manifestations are largely related to the consequences of restrictive ventricular filling, valvular regurgitation, arrhythmias, embolic phenomena and cardiomyopathy, including left- and right-sided heart failure.(I) The prognosis is poor in advanced disease.

Although accurate epidemiologic data are lacking, EMF is estimated to be the most common form of restrictive cardiomyopathy globally. An echocardiographic screening study in Mozambique found a population prevalence of $20 \%$, though

\section{ABSTRACT}

Endomyocardial fibrosis (EMF) is an idiopathic disorder of the tropical and subtropical regions of the world, considered endemic in tropical and subtropical Africa and is characterised by development of restrictive cardiomyopathy. Although accurate epidemiological data are lacking, EMF is estimated to be the most common form of restrictive cardiomyopathy globally. We report on a young patient presenting with a stroke diagnosed with EMF and review the role of cardiovascular magnetic resonance in evaluation of EMF. SAHeart 2020;17:214-216

the study included patients with early, subclinical disease.(2) Clinical presentation is characterised by a bimodal peak at the ages of 10 and 30 years, respectively. (3) Pathologically, there is fibrosis of the RV and/or LV apical endocardial surfaces that leads to restrictive physiology. Furthermore, tethering of the atrioventricular (AV) valve papillary muscles and/or fibrosis of the respective ventricular inflow tracts leads to significant AV valve regurgitation. Gross pathology reveals ventricular endocardial thickening and fibrosis often with overlying thrombus. The atrium of the affected ventricle is often dramatically enlarged, with reduced ventricular volumes. Histopathology demonstrates increased type I collagen deposition, subendocardial infarction, fibrosis, and thrombus. ${ }^{(4)}$ On pathological examination, there is a lack of inflammation or eosinophilia, implying that at end-stage disease, the inflammatory process is inactive. ${ }^{(5)}$

Cardiovascular magnetic resonance (CMR) is well suited to address the early diagnosis, clinical follow up and surgical planning in EMF. Pathological and haemodynamic features of the disease can be identified easily by CMR. These include LV function, presence of thrombus, inflammation, regurgitant valves, diastolic dysfunction and presence of fibrosis (late gadolinium enhancement - LGE).

\section{CASE REPORT}

We report on a 35-year-old man, who was previously well with no medical comorbidity and no prior hospitalisation. He was physically active with an excellent performance status and has never travelled outside of the Western Cape Province. $\mathrm{He}$ is married with I child and employed as a security officer. 

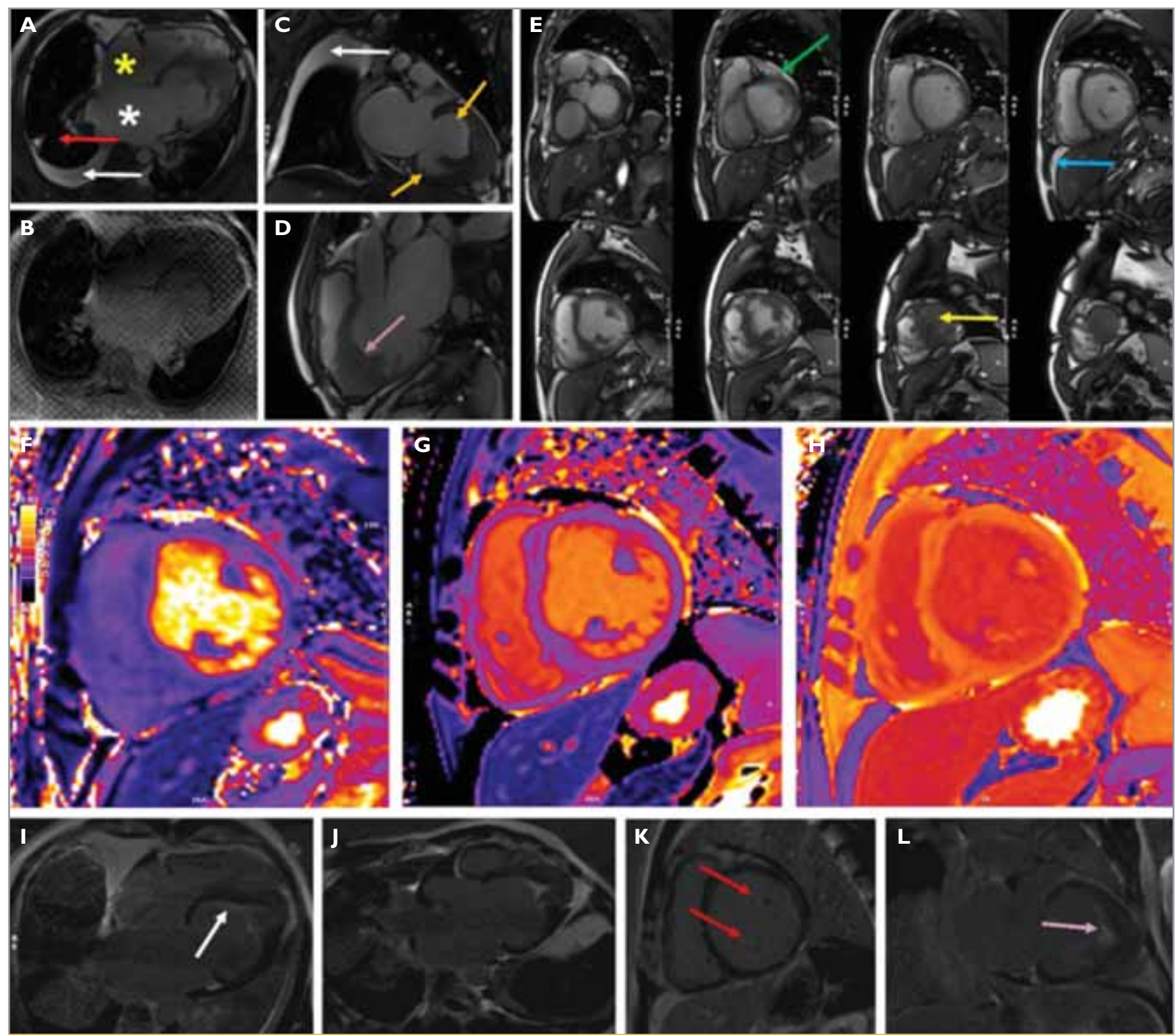

FIGURE I: Cardiovascular magnetic resonance features of endomyocardial fibrosis.

(A) Balanced steady state free precession (bSSFP) horizontal long axis (HLA) view showing dilated left ventricle (LV), biatrial enlargement - left atrium (LA) with white asterix and right atrium (RA) with yellow asterix, obliterated apex with filling defect and thickened, retracted papillary muscles, as well as the classical $V$ sign on top of the apical filling. In addition, there is partial atelectasis of the right lung (red arrow), with bilateral pleural effusions, bigger on the right (white arrow); (B) Cine tagging image showing that the apical filling in the LV apex was adherent to the myocardium and did not move independently, as well as confirming severe diastolic dysfunction and strain impairment; (C) bSSFP vertical long axis view showing the C-shaped basal segments of the LV wall (orange arrows) with a bowl-shaped configuration of the proximal LV, a dilated LA, and a collapsed right lung and a moderately sized right-sided pleural effusion (white arrow); (D) bSSFP left ventricular outflow tract (LVOT) view showing an obliterated apex with a V sign above the filled apex (purple arrow) and a dilated LA; (E) short-axis (SA) stack showing a dilated LV, a small pericardial effusion (green arrow), obliteration of the apex below the papillary muscles (yellow arrow), and a small amount of ascites (blue arrow); (F) T2 mapping showing absence of myocardial oedema/inflammation (T2 46 ms); (G) native TI mapping showing elevated TI time (TI $1376 \mathrm{~ms})$; (H) postcontrast TI image, with extracellular volume value of $37 \%$; (I) LGE HLA image showing subendocardial enhancement (white arrow) and calcified thrombus in the LV apex and the classical bowl-shaped proximal portion of the LV. In addition, the thin walls of the LA also enhance; (J) LGE LVOT view showing a classical V sign in LV apex; (K) LGE (SA) stack showing enhancement of the papillary muscles (red arrows) and a small pericardial effusion; and (L) LGE VLA view showing the classical V sign at the apex and the subendocardial enhancement.

He collapsed while in duty at $02 \mathrm{~h} 00$ in the morning. He claims he did not lose consciousness but was unable to stand because of acute onset weakness in the right arm and leg. He was discovered by his colleagues while lying on the ground and brought by ambulance to the emergency unit at Groote Schuur Hospital. On arrival, his Glasgow Coma Score was 15/15 and he had an obvious left 7th cranial nerve palsy, slurring of his speech and a dense right-sided hemiplegia. He 
was also diagnosed with heart failure. His electrocardiogram showed frequent ventricular ectopy but was otherwise unremarkable and a brain computed tomogram confirmed a left middle cerebral artery infarct. His full blood count and renal function were normal. There was no eosinophilia in his differential white blood cell count. The next morning, he had an echocardiogram that revealed severe LV systolic dysfunction, LV ejection fraction (LVEF) of $30 \%$ and obliteration of his LV apex, with severe diastolic dysfunction and severe mitral and tricuspid regurgitation. A provisional diagnosis of EMF was made, and he was referred for CMR.

CMR (Figure I) confirmed mildly increased LV volumes (LV EDV 208mL; LVESV $144 \mathrm{~mL}$ ). He had severely impaired LV systolic function (LVEF 31\%) and marked global hypokinesia of all LV segments. There was no LV hypertrophy. The apex was akinetic and there was a filling defect in the apex of the LV. The tissue characteristics were in keeping with calcified thrombus. The LV walls had normal thickness. There was marked increased left atrial size $(66 \mathrm{~mm}$ in the 3 -chamber view; area $\left.74.8 \mathrm{~cm}^{2}\right)$. There were intact interventricular and interatrial septae. He had severe mitral regurgitation, with a mitral valve that opened normally, and with thin mobile leaflets. There was a tricuspid aortic valve, which opened normally, with mild aortic regurgitation and normal aortic dimensions. The RV volumes were mildly increased, with moderate impairment in RV systolic function (RVEF 39\%). There was no RV hypertrophy. The right atrium was moderately increased (area $36.3 \mathrm{~cm}^{2}$ ). He had normal tricuspid valve morphology and severe tricuspid regurgitation and a central jet of mild pulmonic regurgitation. He had a mildly dilated right ventricular outflow tract $(31 \mathrm{~mm})$ and a dilated pulmonary artery $(38 \mathrm{~mm})$. On TI- and T2-weighted images, there was no myocardial oedema. On parametric mapping, he had a normal T2 time (46ms), but an elevated native TI time (I 376ms) as well as an elevated extracellular volume fraction (37\%). On LGE imaging, there was subendocardial enhancement of the LV, with large apical thrombus that enhanced in the classical $V$ shape, and fine, patchy enhancement in the myocardium and apex. Of note, the thin walls of the dilated left atrium also took up contrast. Both papillary muscles also enhanced. There was no RV enhancement. The pericardium was normal in thickness with a small effusion ( $12 \mathrm{~mm})$. The lungs showed moderate bilateral pleural effusions, partial collapse of the right lung and bilateral pulmonary interstitial infiltration in keeping with pulmonary oedema and upper lobe venous diversion.

The patient was treated for heart failure and had almost complete resolution of his neurological deficit. His Holter monitoring has failed to show atrial fibrillation or atrial flutter. He continues to work as a security guard and follows up at the cardiomyopathy specialist clinic.

\section{DISCUSSION}

CMR lends itself to comprehensive evaluation of EMF. Balanced steady state free precession imaging is ideal for assessment of LV and RV volumes, mass and function as well as pleural and pericardial effusions and the presence of extra-cardiac masses. In addition, these sequences are useful for the evaluation of typical features of EMF including biatrial dilatation, a $\vee$ sign (cased by layered thrombus) in the apex, filling and obliteration of the LV (and RV) apex, and basal segments of the LV that appear like a bowl (or 2 C's facing each other). Parametric mapping may be used to assess for acute myocardial oedema or inflammation in the acute phase as well as the presence of diffuse fibrosis and expanded interstitial matrix. LGE imaging is important for assessment of focal fibrosis in the myocardium, as well as a typical subendocardial pattern or so-called Zebra pattern secondary to layered overlying thrombus. The fibrotic may extend to the subvalvular plane, with involvement of papillary muscles, and retraction of the apex. Regurgitant lesions of the valves, as in this case, are common and occur because of fibrosis of the subvalvular apparatus causing restriction of the posterior mitral leaflet on the left side and the posterior aspect of the tricuspid valve on the right side. ${ }^{(6-8)}$

\section{CONCLUSIONS}

CMR is a useful imaging tool for diagnosis of EMF, for risk stratification, and for planning of surgery and may be used at every stage of the disease.

\section{Conflict of interest: none declared.}

\section{REFERENCES}

I. Sliwa K, Damasceno A, Mayosi BM. Epidemiology and etiology of cardiomyopathy in Africa. Circulation 2005; 1 I 2(23):3577-3583.

2. Mocumbi $A O$, Ferreira MB, Sidi D, et al. A population study of endomyocardial fibrosis in a rural area of Mozambique. N Engl J Med 2008; 359( 1$): 43-49$

3. Rutakingirwa M, Ziegler JL, Newton R, et al. Poverty and eosinophilia are risk factors for endomyocardial fibrosis (EMF) in Uganda. Trop Med Int Health 1999:4(3):229-235.

4. Mocumbi AO, Yacoub MH, Yokohama $\mathrm{H}$, et al. Right ventricular endomyocardial fibrosis. Cardiovasc Pathol 2009; 18(1):64-65.

5. Grimaldi A, Mocumbi AO, Freers J, et al. Tropical endomyocardial fibrosis: Natural history, challenges, and perspectives. Circulation 2016; 133(24):2503-2315.

6. Salemi VM, Rochitte CE, Shiozaki AA, et al. Late gadolinium enhancement magnetic resonance imaging in the diagnosis and prognosis of endomyocardial fibrosis patients. Circ Cardiovasc Imaging 201 1;4(3):304-31 I.

7. Leon D, Martin M, Corros C, et al. Usefulness of cardiac MRI in the early diagnosis of endomyocardial fibrosis. Rev Port Cardiol 20 I 2;3 I (5):40 I-402.

8. Carneiro AC. A new typical finding in late gadolinium enhanced images for the diagnosis of endomyocardial fibrosis - the double $V$ sign. J Cardiovasc Magn Reson 201 I ( 3(Suppl I):O40. 